MATHEMATICS OF COMPUTATION

Volume 66, Number 217, January 1997, Pages 345-361

S 0025-5718(97)00786-2

\title{
THE QUASI-LAGUERRE ITERATION
}

\author{
QIANG DU, MING JIN, T.Y. LI, AND Z. ZENG
}

\begin{abstract}
The quasi-Laguerre iteration has been successfully established, by the same authors, in the spirit of Laguerre's iteration for solving the eigenvalues of symmetric tridiagonal matrices. The improvement in efficiency over Laguerre's iteration is drastic. This paper supplements the theoretical background of this new iteration, including the proofs of the convergence properties.
\end{abstract}

\section{INTRODUCTION}

When the Laguerre iteration [4]

$$
L_{ \pm}(x)=x+\frac{n}{\left(-\frac{f^{\prime}(x)}{f(x)}\right) \pm \sqrt{(n-1)\left[(n-1)\left(-\frac{f^{\prime}(x)}{f(x)}\right)^{2}-n\left(\frac{f^{\prime \prime}(x)}{f(x)}\right)\right]}}
$$

is used to solve a polynomial $f$ with all its zeros being real, such as the characteristic polynomial of a real symmetric matrix, the most important advantages are its global and monotonic convergence. While its ultimate convergence rate is cubic, the requirement of evaluating $f^{\prime \prime}$, which is relatively time consuming, constitutes a major disadvantage of this iteration in terms of its efficiency. A new iteration, which we called the quasi-Laguerre iteration, has been established in [1] which avoids the evaluation of $f^{\prime \prime}$ but still maintains global and monotone convergence when applied to polynomials with all real zeros. The purpose of this paper is to supplement the theoretical background of this new iteration.

Formula (1.1) can be derived in diverse ways. The best one seems to be to answer the following question [3]:

Question 1. Among all polynomials $p(x)$ of degree $n$ with $n$ real zeros and with $p\left(x_{0}\right)=f\left(x_{0}\right) \neq 0, p^{\prime}\left(x_{0}\right)=f^{\prime}\left(x_{0}\right)$ and $p^{\prime \prime}\left(x_{0}\right)=f^{\prime \prime}\left(x_{0}\right)$ at a specified real $x_{0}$, which one has a zero closest to $x_{0}$ ? and where?

In general, of all those polynomials, $L_{+}\left(x_{0}\right)$ in (1.1) gives the closest zero from the right and $L_{-}\left(x_{0}\right)$ gives the closest one from the left.

To avoid the evaluation of $f^{\prime \prime}$, the above optimization problem can be revised as follows:

Received by the editor August 9, 1995 and, in revised form, September 15, 1995.

1991 Mathematics Subject Classification. Primary 65F15; Secondary 65F40.

The research of the first author was supported in part by NSF under Grant DMS-9500718.

The research of the third author was supported in part by NSF under Grant DMS-9504953 and by a Guggenheim Fellowship.

(C)1997 American Mathematical Society 
Question 2. Given two specified reals $a<b$, among all polynomials $p(x)$ of degree $n$ with $n$ real zeros, none of which lie in $[a, b]$, and with

$$
\frac{p^{\prime}(a)}{p(a)}=\frac{f^{\prime}(a)}{f(a)} \text { and } \frac{p^{\prime}(b)}{p(b)}=\frac{f^{\prime}(b)}{f(b)},
$$

which one has a zero closest to $a$ from the right or from the left? and where?

Even further, to account for multiple zeros, we reformulate Question 2 in a more general form:

Question 3. Given two specified reals $a<b$, for polynomials satisfying the conditions in Question 2, consider their $m$ th $(m<n)$ zero to the right (or to the left) of $a$. Which polynomial has the closest one to $a$ from the right (or from the left)? and where?

This optimization problem has been solved in [1] and the solution has a closed form. To convert the solution of this optimization problem to an iterative scheme, let $z_{M}$ be a zero of $f$ with multiplicity $M \geq m$. To approximate $z_{M}$ from its left, suppose $z_{M}$ is on the right-hand side of two starting points $x_{m}^{(0)}<x_{m}^{(1)}$ and none of the zeros of $f$ lie between $x_{m}^{(0)}$ and $z_{M}$. Then, for $k \geq 1$, we may let $a=x_{m}^{(k-1)}, b=x_{m}^{(k)}$ and let $x_{m}^{(k+1)}$ be the closest $m$ th zero to the right of $x_{m}^{(k)}$ of polynomials satisfying the conditions in Question 2. Similarly, when two starting points $x_{m}^{(1)}<x_{m}^{(0)}$ are available on the right-hand side of $z_{M}$ with no zeros of $f$ lying in $\left(z_{M}, x_{m}^{(0)}\right)$, then for $k \geq 1$, we let $a=x_{m}^{(k)}, b=x_{m}^{(k-1)}$ and let $x_{m}^{(k+1)}$ be the closest $m$ th zero to the left of $x_{m}^{(k)}$ of polynomials satisfying the conditions in Question 2.

The sequence $\left\{x_{m}^{(k)}\right\}$ so constructed is obviously a monotone sequence. It will be shown in $\S 3$ that this sequence converges to $z_{M}$ with ultimate convergence rate $\sqrt{2}+$ 1 when $m=M$, and the convergence is linear if $m<M$. The linear convergence ratio,

will also be given.

$$
\lim _{k \rightarrow \infty} \frac{x_{m}^{(k+1)}-z_{M}}{x_{m}^{(k)}-z_{M}},
$$

In practice, the multiplicity of any zero of $f$ is, in general, not revealed ahead of time. Therefore, we can only use $m=1$ in our iterations. We will show in $\S 4$ that when a multiple zero occurs and when the linear convergence ratio becomes evident, the convergence can be speeded up substantially by a special device.

This newly derived quasi-Laguerre iteration was employed to approximate eigenvalues of symmetric tridiagonal matrices with remarkable results. The details of the practical implementation and comprehensive numerical experiments on diverse types of matrices can be found in [1]. Our algorithm considerably improves the speed of Laguerre's iteration for the same purpose [1].

During the writing of this paper, we became aware of an earlier unpublished work of L. Foster [2] in which a class of globally convergent iterations, including our quasi-Laguerre iteration for the case of simple roots, were studied. Our work here is based on a different approach and achieves much more general results.

\section{The QuASi-Laguerre ITERATION}

For the reader's convenience, we briefly outline the basic algorithm for the quasiLaguerre iteration in this section. A more detailed derivation can be found in [1] 
and we only introduce the relevant part that is essential for the presentation of the convergence analysis given in $\S 3$.

We are given a polynomial $f$ of degree $n$ with all of its zeros being real. For $a<b$ with $f(a) f(b) \neq 0$, let $\mathcal{F}$ be the class of polynomials $p(x)$ of degree $n$ that satisfy the following conditions:

(i) all zeros of $p(x)$ are real;

(ii) none of the zeros of $p(x)$ are in $[a, b]$;

(iii) $p(a) p(b) \neq 0$ and

$$
\frac{p^{\prime}(a)}{p(a)}=\frac{f^{\prime}(a)}{f(a)} \equiv q(a), \quad \frac{p^{\prime}(b)}{p(b)}=\frac{f^{\prime}(b)}{f(b)} \equiv q(b) .
$$

In answering Question 3, stated in $\S 1$, we are led to the quadratic equation [1]

$$
m r(b, a) Y^{2}-\left[r(a, b) r(b, a)-n^{2}+2 m n\right] Y+m r(a, b)=0,
$$

where

$$
r(a, b) \equiv n+(b-a) q(a) \quad \text { and } \quad r(b, a)=n+(a-b) q(b) .
$$

Its solutions are given by

$$
Y_{m \pm}=\frac{r(a, b) r(b, a)-n^{2}+2 m n \pm \sqrt{\left[r(a, b) r(b, a)-n^{2}\right]\left[r(a, b) r(b, a)-(n-2 m)^{2}\right]}}{2 m r(b, a)} .
$$

Now, as in [1], let

$$
\begin{aligned}
& u_{m \pm}=a-\frac{b-a}{Y_{m \mp}-1}=a- \\
& \frac{2 m r(b, a)(b-a)}{r(a, b) r(b, a)-n^{2}+2 m n-2 m r(b, a) \mp \sqrt{\left[r(a, b) r(b, a)-n^{2}\right]\left[r(a, b) r(b, a)-(n-2 m)^{2}\right]}} \\
& \quad=a+\frac{2 m[n-(b-a) q(b)]}{-(b-a) R-2 m q(b) \pm \sqrt{R\left[(b-a)^{2} R+4 m(n-m)\right]}},
\end{aligned}
$$

where

and also define

$$
R=n\left(\frac{q(a)-q(b)}{b-a}\right)-q(a) q(b)
$$

The solutions of the optimization problems in Question 3 can then be described as follows (see [1] for details):

(i) If $f$ has at least $m$ (counting multiplicities) zeros to the left of $a$, then the zero $u_{m-}$ in (2.4) of the polynomial

$$
p_{1}(x)=C\left(x-u_{m-}\right)^{m}\left(x-v_{1-}\right)^{n-m}
$$

is the closest $m$ th zero to the left of $a$ among all polynomials in $\mathcal{F}$. And it is clear that

$$
z_{m-} \leq u_{m-} \leq \cdots \leq u_{2-} \leq u_{1-}<a<b,
$$

where $z_{m-}$ is the $m$ th zero of $f$ to the left of $a$. 
(ii) If $f$ has at least $m$ (counting multiplicities) zeros to the right of $b$, then the zero $u_{m+}$ in (2.4) of the polynomial

$$
p_{2}(x)=C\left(x-u_{m+}\right)^{m}\left(x-v_{1+}\right)^{n-m}
$$

is the closest $m$ th zero to the right of $b$ among all polynomials in $\mathcal{F}$. It is also clear that

$$
a<b<u_{1+} \leq u_{2+} \leq \cdots \leq u_{m+} \leq z_{m+},
$$

where $z_{m+}$ is the $m$ th zero of $f$ to the right of $b$.

Let a polynomial $f$ of degree $n$ be given with all its zeros being real. Let $z_{M}$ be a zero of $f$ with multiplicity $M \geq 1$. To approximate $z_{M}$ from its left, we shall use $u_{m+}$ in (2.4) with $m \leq M$ to generate a monotonically increasing sequence $\left\{x_{m+}^{(k)}\right\}$ which converges to $z_{M}$ as $k \rightarrow \infty$. Similarly, to approximate $z_{M}$ from its right, a monotonically decreasing sequence $\left\{x_{m-}^{(k)}\right\}$ converging to $z_{M}$, as $k \rightarrow \infty$, can be generated by using $u_{m-}$ in (2.4).

To be more precise, suppose $z_{M}$ is on the right-hand side of two starting points $x_{m+}^{(0)}<x_{m+}^{(1)}$ and none of the zeros of $f$ lie between $x_{m+}^{(0)}$ and $z_{M}$. Then, for $k \geq 1$, we let

$$
\begin{aligned}
& x_{m+}^{(k+1)}=x_{m+}^{(k)}+ \\
& \left.-\left(x_{m+}^{(k-1)}-x_{m+}^{(k)}\right) R-2 m q\left(x_{m+}^{(k-1)}\right)+\sqrt{R\left[\left(x_{m+}^{(k-1)}-x_{m+}^{(k)}\right)^{2} R+4 m(n-m)\right.}\right]
\end{aligned}
$$

where

$$
q\left(x_{m+}^{(k)}\right)=\frac{f^{\prime}\left(x_{m+}^{(k)}\right)}{f\left(x_{m+}^{(k)}\right)}, \quad q\left(x_{m+}^{(k-1)}\right)=\frac{f^{\prime}\left(x_{m+}^{(k-1)}\right)}{f\left(x_{m+}^{(k-1)}\right)}
$$

and

$$
R=n\left(\frac{q\left(x_{m+}^{(k-1)}\right)-q\left(x_{m+}^{(k)}\right)}{x_{m+}^{(k)}-x_{m+}^{(k-1)}}\right)-q\left(x_{m+}^{(k-1)}\right) q\left(x_{m+}^{(k)}\right) .
$$

In other words, we replace $u_{m+}, a$ and $b$ in (2.4) by $x_{m+}^{(k+1)}, x_{m+}^{(k)}$ and $x_{m+}^{(k-1)}$, respectively.

From what has been presented earlier (and derived in more detail in [1]), it is easy to see that

$$
x_{m+}^{(k)}<x_{m+}^{(k+1)} \leq z_{M}
$$

and no zeros of $f$ fall between $x_{m+}^{(k+1)}$ and $z_{M}$. So the sequence $\left\{x_{m+}^{(k)}\right\}$ satisfies

$$
x_{m+}^{(0)}<x_{m+}^{(1)}<\cdots<x_{m+}^{(k)}<\cdots \leq z_{M} .
$$

Similarly, when two starting points $x_{m-}^{(1)}<x_{m-}^{(0)}$ are available on the right-hand side of $z_{M}$ with no zeros of $f$ lying in $\left(z_{M}, x_{m-}^{(0)}\right)$, then for $k \geq 1$, let $a=x_{m-}^{(k)}$, 
$b=x_{m-}^{(k-1)}$ and $u_{m-}=x_{m-}^{(k+1)}$ in (2.4). Namely,

$$
\begin{aligned}
& x_{m-}^{(k+1)}=x_{m-}^{(k)}+ \\
& \frac{2 m\left[n-\left(x_{m-}^{(k-1)}-x_{m-}^{(k)}\right) q\left(x_{m-}^{(k-1)}\right)\right]}{-\left(x_{m-}^{(k-1)}-x_{m-}^{(k)}\right) R-2 m q\left(x_{m-}^{(k-1)}\right)-\sqrt{R\left[\left(x_{m-}^{(k-1)}-x_{m-}^{(k)}\right)^{2} R+4 m(n-m)\right.}},
\end{aligned}
$$

where

$$
q\left(x_{m-}^{(k)}\right)=\frac{f^{\prime}\left(x_{m-}^{(k)}\right)}{f\left(x_{m-}^{(k)}\right)}, \quad q\left(x_{m-}^{(k-1)}\right)=\frac{f^{\prime}\left(x_{m-}^{(k-1)}\right)}{f\left(x_{m-}^{(k-1)}\right)}
$$

and

$$
R=n\left(\frac{q\left(x_{m-}^{(k)}\right)-q\left(x_{m-}^{(k-1)}\right)}{x_{m-}^{(k-1)}-x_{m-}^{(k)}}\right)-q\left(x_{m-}^{(k)}\right) q\left(x_{m-}^{(k-1)}\right)
$$

Again it is clear that

$$
z_{M} \leq x_{m-}^{(k+1)}<x_{m-}^{(k)}
$$

and none of the zeros of $f$ lie between $z_{M}$ and $x_{m-}^{(k+1)}$. Moreover, the sequence $\left\{x_{m-}^{(k)}\right\}$ satisfies

$$
z_{M} \leq \cdots<x_{m-}^{(k)}<\cdots<x_{m-}^{(1)}<x_{m-}^{(0)}
$$

We call the process of generating the sequences $\left\{x_{m \pm}^{(k)}\right\}$ defined by (2.10) and (2.11) the quasi-Laguerre iterations.

\section{Convergence AnAlysis}

We first prove the following theorem.

Theorem 3.1. Both sequences $\left\{x_{m \pm}^{(k)}\right\}$ converge to $z_{M}$ monotonically.

Proof. We will prove the theorem for the sequence $\left\{x_{m-}^{(k)}\right\}_{k=1}^{\infty}$. Similar arguments hold for the sequence $\left\{x_{m+}^{(k)}\right\}_{k=1}^{\infty}$. We shall write $y_{k}$ for $x_{m-}^{(k)}$ when there is no ambiguity. Obviously, by the way it is constructed, $\left\{y_{k}\right\}$ is a decreasing sequence bounded below by $z_{M}$. Then it must converge to a certain number $x^{*} \geq z_{M}$. Let $z^{(1)}, \ldots, z^{(n-M)}$ be the rest of the zeros of $f$ besides $z_{M}$. Suppose $x^{*} \neq z_{M}$; then $f\left(x^{*}\right) \neq 0$. Recall that

$$
q(y)=\frac{f^{\prime}(y)}{f(y)}=\sum_{i=1}^{n-M} \frac{1}{y-z^{(i)}}+\frac{M}{y-z_{M}} .
$$


Thus,

$$
\begin{aligned}
R= & n\left(\frac{q\left(y_{k}\right)-q\left(y_{k-1}\right)}{y_{k-1}-y_{k}}\right)-q\left(y_{k}\right) q\left(y_{k-1}\right) \\
= & \frac{n}{y_{k-1}-y_{k}}\left[\sum_{i=1}^{n-M} \frac{1}{y_{k}-z^{(i)}}+\frac{M}{y_{k}-z_{M}}-\sum_{i=1}^{n-M} \frac{1}{y_{k-1}-z^{(i)}}-\frac{M}{y_{k-1}-z_{M}}\right] \\
& -q\left(y_{k}\right) q\left(y_{k-1}\right) \\
= & n\left[\sum_{i=1}^{n-M} \frac{1}{\left(y_{k}-z^{(i)}\right)\left(y_{k-1}-z^{(i)}\right)}+\frac{M}{\left(y_{k}-z_{M}\right)\left(y_{k-1}-z_{M}\right)}\right]-q\left(y_{k}\right) q\left(y_{k-1}\right) \\
\rightarrow & n\left[\sum_{i=1}^{n-M} \frac{1}{\left(x^{*}-z^{(i)}\right)^{2}}+\frac{M}{\left(x^{*}-z_{M}\right)^{2}}\right]-\left[q\left(x^{*}\right)\right]^{2} \equiv \hat{R}<\infty .
\end{aligned}
$$

From (2.11),

$$
y_{k+1}=y_{k}-\frac{2 m\left[n-\left(y_{k-1}-y_{k}\right) q\left(y_{k-1}\right)\right]}{\left.\left(y_{k-1}-y_{k}\right) R+2 m q\left(y_{k-1}\right)+\sqrt{R\left[\left(y_{k-1}-y_{k}\right)^{2} R+4 m(n-m)\right.}\right]} .
$$

Taking $y_{k} \longrightarrow x^{*}$, we have

$$
x^{*}=x^{*}-\frac{2 m n}{2 m q\left(x^{*}\right)+\sqrt{4 m(n-m) \hat{R}}} \neq x^{*},
$$

which leads to a contradiction.

The next theorem provides the rate of convergence of both sequences $\left\{x_{m \pm}^{(k)}\right\}$.

Theorem 3.2. (i) When $m<M$, the convergence of $\left\{x_{m \pm}^{(k)}\right\}$ to $z_{M}$ is linear and the convergence ratio

$$
\omega \equiv \lim _{k \rightarrow \infty} \frac{x_{m \pm}^{(k+1)}-z_{M}}{x_{m \pm}^{(k)}-z_{M}}
$$

is the only real solution in $(0,1)$ of

$$
\frac{n-M-m}{n-M} x^{3}-x^{2}-x+\frac{M-m}{M}=0 .
$$

(ii) When $m=M$, the convergence of $\left\{x_{m \pm}^{(k)}\right\}$ to $z_{M}$ is superlinear with convergence rate $\sqrt{2}+1$.

Proof. Again, we will prove the theorem for the sequence $\left\{x_{m-}^{(k)}\right\}_{k=1}^{\infty}$ and write $y_{k}$ for $x_{m-}^{(k)}$ when there is no ambiguity. Similar arguments hold for the sequence $\left\{x_{m+}^{(k)}\right\}_{k=1}^{\infty}$.

From (2.11),

$$
y_{k+1}=y_{k}-\frac{2 m\left[n-\left(y_{k-1}-y_{k}\right) q\left(y_{k-1}\right)\right]}{\left(y_{k-1}-y_{k}\right) R+2 m q\left(y_{k-1}\right)+\sqrt{R\left[\left(y_{k-1}-y_{k}\right)^{2} R+4 m(n-m)\right]}},
$$

where

$$
q\left(y_{k-1}\right)=\frac{f^{\prime}\left(y_{k-1}\right)}{f\left(y_{k-1}\right)}, \quad q\left(y_{k}\right)=\frac{f^{\prime}\left(y_{k}\right)}{f\left(y_{k}\right)}, \quad \text { and }
$$




$$
R=n\left(\frac{q\left(y_{k}\right)-q\left(y_{k-1}\right)}{y_{k-1}-y_{k}}\right)-q\left(y_{k}\right) q\left(y_{k-1}\right) .
$$

Let $z^{(1)}, \ldots, z^{(n-M)}$ be the rest of the zeros of $f$ besides $z_{M}$. Then,

$$
\begin{aligned}
q\left(y_{k-1}\right) & =\frac{f^{\prime}\left(y_{k-1}\right)}{f\left(y_{k-1}\right)}=\frac{M}{y_{k-1}-z_{M}}+Q\left(y_{k-1}\right) \quad \text { with } \quad Q\left(y_{k-1}\right) \equiv \sum_{i=1}^{n-M} \frac{1}{y_{k-1}-z^{(i)}}, \\
q\left(y_{k}\right) & =\frac{f^{\prime}\left(y_{k}\right)}{f\left(y_{k}\right)}=\frac{M}{y_{k}-z_{M}}+Q\left(y_{k}\right) \quad \text { with } \quad Q\left(y_{k}\right) \equiv \sum_{i=1}^{n-M} \frac{1}{y_{k}-z^{(i)}}
\end{aligned}
$$

and

$$
\begin{aligned}
R & =n\left(\frac{q\left(y_{k}\right)-q\left(y_{k-1}\right)}{y_{k-1}-y_{k}}\right)-q\left(y_{k}\right) q\left(y_{k-1}\right) \\
& =\frac{M(n-M)}{\left(y_{k}-z_{M}\right)\left(y_{k-1}-z_{M}\right)}-\frac{M Q\left(y_{k-1}\right)}{y_{k}-z_{M}}-\frac{M Q\left(y_{k}\right)}{y_{k-1}-z_{M}}-Q\left(y_{k}\right) Q\left(y_{k-1}\right)+n R_{1}
\end{aligned}
$$

with

$$
R_{1} \equiv \sum_{i=1}^{n-M} \frac{1}{\left(y_{k-1}-z^{(i)}\right)\left(y_{k}-z^{(i)}\right)} .
$$

Since $y_{k} \rightarrow z_{M}$ as $k \rightarrow \infty$, we have

$$
\begin{aligned}
Q\left(y_{k-1}\right) & =\sum_{i=1}^{n-M} \frac{1}{y_{k-1}-z^{(i)}} \rightarrow Q \equiv \sum_{i=1}^{n-M} \frac{1}{z_{M}-z^{(i)}}<\infty \\
Q\left(y_{k}\right) & =\sum_{i=1}^{n-M} \frac{1}{y_{k}-z^{(i)}} \rightarrow Q
\end{aligned}
$$

and

$$
R_{1}=\sum_{i=1}^{n-M} \frac{1}{\left(y_{k-1}-z^{(i)}\right)\left(y_{k}-z^{(i)}\right)} \rightarrow R^{*} \equiv \sum_{i=1}^{n-M} \frac{1}{\left(z_{M}-z^{(i)}\right)^{2}}<\infty .
$$

Let $\epsilon_{k}=\frac{y_{k}-z_{M}}{y_{k-1}-z_{M}}$ for all $k \geq 1$; then $\frac{y_{k-1}-y_{k}}{y_{k-1}-z_{M}}=1-\epsilon_{k}$. Obviously, $0<\epsilon_{k}<1$ and $0<1-\epsilon_{k}<1$. From (3.1),

$$
\frac{\frac{y_{k+1}-z_{M}}{y_{k}-z_{M}}=1-}{\left(y_{k}-z_{M}\right)\left\{\left(y_{k-1}-y_{k}\right) R+2 m q\left(y_{k-1}\right)+\sqrt{R\left[\left(y_{k-1}-y_{k}\right)^{2} R+4 m(n-m)\right.}\right]} .
$$

Now,

$$
\begin{aligned}
\text { (a) } & \left(y_{k-1}-y_{k}\right) q\left(y_{k-1}\right)=\left(y_{k-1}-y_{k}\right)\left[\frac{M}{y_{k-1}-z_{M}}+Q\left(y_{k-1}\right)\right] \\
= & \left(y_{k-1}-z_{M}\right)\left[1-\frac{y_{k}-z_{M}}{y_{k-1}-z_{M}}\right]\left[\frac{M}{y_{k-1}-z_{M}}+Q\left(y_{k-1}\right)\right] \\
= & \left(1-\epsilon_{k}\right)\left[M+Q\left(y_{k-1}\right)\left(y_{k-1}-z_{M}\right)=M\left(1-\epsilon_{k}\right)+O\left(y_{k-1}-z_{M}\right),\right. \\
\text { (b) } & \left(y_{k}-z_{M}\right)\left(y_{k-1}-z_{M}\right) R=M(n-M)-Q\left(y_{k}\right) Q\left(y_{k-1}\right)\left(y_{k}-z_{M}\right)\left(y_{k-1}-z_{M}\right) \\
& -\left(y_{k-1}-z_{M}\right) M Q\left(y_{k-1}\right)-\left(y_{k}-z_{M}\right) M Q\left(y_{k}\right)+n\left(y_{k}-z_{M}\right)\left(y_{k-1}-z_{M}\right) R_{1} \\
= & M(n-M)+O\left(y_{k-1}-z_{M}\right),
\end{aligned}
$$


(c) $\quad\left(y_{k}-z_{M}\right)\left(y_{k-1}-y_{k}\right) R=\left(1-\epsilon_{k}\right)\left(y_{k}-z_{M}\right)\left(y_{k-1}-z_{M}\right) R$ $=\left(1-\epsilon_{k}\right) M(n-M)+O\left(y_{k-1}-z_{M}\right)$ (because of (b) above),

(d) $\quad\left(y_{k}-z_{M}\right)^{2} R=\epsilon_{k}\left(y_{k}-z_{M}\right)\left(y_{k-1}-z_{M}\right) R=\epsilon_{k} M(n-M)+O\left(y_{k-1}-z_{M}\right)$ (because of (b) above),

(e) $\left(y_{k}-z_{M}\right)^{2} R\left[\left(y_{k-1}-y_{k}\right)^{2} R+4 m(n-m)\right]$ $=\left[\left(y_{k}-z_{M}\right)\left(y_{k-1}-y_{k}\right) R\right]^{2}+4 m(n-m)\left(y_{k}-z_{M}\right)^{2} R$ $=\left(1-\epsilon_{k}\right)^{2} M^{2}(n-M)^{2}+4 m(n-m) \epsilon_{k} M(n-M)+O\left(y_{k-1}-z_{M}\right)$ (because of (c) and (d) above).

Substituting (a), (c), (e) into (3.3) yields

$$
\begin{aligned}
& \epsilon_{k+1}=\frac{y_{k+1}-z_{M}}{y_{k}-z_{M}}=1- \\
& \frac{2 m\left[n-\left(1-\epsilon_{k}\right) M\right]+O\left(y_{k-1}-z_{M}\right)}{M(n-M)\left(1-\epsilon_{k}\right)+2 m M \epsilon_{k}+M(n-M) \sqrt{\left(1-\epsilon_{k}\right)^{2}+\frac{4 m(n-m)}{M(n-M)} \epsilon_{k}}+O\left(y_{k-1}-z_{M}\right)} \\
& \quad=G\left(\epsilon_{k}\right)+O\left(y_{k-1}-z_{M}\right),
\end{aligned}
$$

where

$$
G(\epsilon)=1-\frac{2 m[n-(1-\epsilon) M]}{M(n-M)(1-\epsilon)+2 m M \epsilon+M(n-M) \sqrt{(1-\epsilon)^{2}+\frac{4 m(n-m)}{M(n-M)} \epsilon}} .
$$

We claim that $G(\epsilon)$ can be rewritten as

$$
G(\epsilon)=\frac{2(M-m)}{M\left(1+\epsilon+\sqrt{(1-\epsilon)^{2}+\frac{4 m(n-m)}{M(n-M)}} \epsilon\right)} .
$$

To see this, let $\triangle=\sqrt{(1-\epsilon)^{2}+\frac{4 m(n-m)}{M(n-M)} \epsilon}$ and

$$
\begin{aligned}
& A=m[n-(1-\epsilon) M], \\
& B=(n-M)(1-\epsilon)+2 m \epsilon+(n-M) \triangle, \\
& C=M-m, \\
& D=(1+\epsilon)+\triangle .
\end{aligned}
$$

Then, $G(\epsilon)=1-\frac{2 A}{M B}$, and

$$
\begin{aligned}
& A D=m(n-M)(1+\epsilon)+m M \epsilon(1+\epsilon)+[m(n-M)+m M \epsilon] \triangle, \\
& B C=(M-m)(n-M)(1-\epsilon)+2(M-m) m \epsilon+(M-m)(n-M) \triangle .
\end{aligned}
$$

So,

$$
\begin{aligned}
A D+B C= & m M \epsilon^{2}+\left(M^{2}-M n+2 m n+M m-2 m^{2}\right) \epsilon \\
& +M(n-M)+[M(n-M)+m M \epsilon] \triangle .
\end{aligned}
$$


Meanwhile,

$$
\begin{aligned}
M B D= & M(n-M)\left(1-\epsilon^{2}\right)+2 m M \epsilon+2 m M \epsilon^{2}+M(n-M)(1-\epsilon)^{2} \\
& +4 m(n-m) \epsilon+[M(n-M)(1+\epsilon)+M(n-M)(1-\epsilon)+2 m M \epsilon] \triangle \\
= & 2 m M \epsilon^{2}+\left(2 M^{2}-2 M n+4 m n+2 M m-4 m^{2}\right) \epsilon \\
& +2 M(n-M)+[2 M(n-M)+2 m M \epsilon] \triangle \\
= & 2(A D+B C) .
\end{aligned}
$$

Thus,

$$
\frac{1}{2} M[1-G(\epsilon)]=\frac{A}{B}=\frac{A}{B}+\frac{C}{D}-\frac{C}{D}=\frac{A D+B C}{B D}-\frac{C}{D}=\frac{M}{2}-\frac{C}{D} .
$$

Therefore,

$$
G(\epsilon)=\frac{2 C}{M D}=\frac{2(M-m)}{M\left(1+\epsilon+\sqrt{(1-\epsilon)^{2}+\frac{4 m(n-m)}{M(n-M)} \epsilon}\right)} .
$$

In this form, it can be easily shown that $G(\epsilon)$ is monotonically decreasing. To prove this, let

$$
h(\epsilon)=1+\epsilon+\sqrt{(1-\epsilon)^{2}+4 \gamma \epsilon}, \quad \text { where } \quad \gamma=\frac{m(n-m)}{M(n-M)} .
$$

For $\epsilon \in(0,1)$,

$$
h^{\prime}(\epsilon)=1+\frac{\epsilon-1+2 \gamma}{\sqrt{(1-\epsilon)^{2}+4 \gamma \epsilon}}>0,
$$

so, from (3.5), $G(\epsilon)$ is monotonically decreasing.

Now,

(i) For $m<M$, let

$$
\beta=\frac{M-m}{M} \in(0,1) \text { and } \gamma=\frac{m(n-m)}{M(n-M)}>0 .
$$

Since $G(\epsilon)$ is monotonically decreasing for $\epsilon \in[0,1]$ and $\epsilon_{k} \in(0,1)$ for all $k \geq 1$, we have

$$
0<\frac{\beta}{1+\sqrt{\gamma}}=G(1)<G\left(\epsilon_{k}\right)<G(0)=\beta<1 .
$$

Hence, when $k$ is sufficiently large,

$$
0<\frac{G(1)}{2}<\epsilon_{k+1}=G\left(\epsilon_{k}\right)+O\left(y_{k-1}-z_{M}\right)<\frac{G(0)+1}{2}<1 .
$$

Consequently, the convergence of $\left\{y_{k}\right\}_{k=1}^{\infty}$ to $z_{M}$ is linear.

Moreover, (3.4) implies that

$$
\left|\epsilon_{k+1}-G\left(\epsilon_{k}\right)\right|=\left|O\left(y_{k-1}-z_{M}\right)\right| \leq C\left(y_{k-1}-z_{M}\right) \quad \text { for } k \geq 1
$$

for some positive number $C$ independent of $k$. Since $y_{k} \rightarrow z_{M}$, there exists an integer $N>1$ such that

$$
G(1)-C\left(y_{N-1}-z_{M}\right)>0 \quad \text { and } \quad G(0)+C\left(y_{N-1}-z_{M}\right)<1 .
$$

Let

$$
c_{1}=G(1)-C\left(y_{N-1}-z_{M}\right), \quad d_{1}=G(0)+C\left(y_{N-1}-z_{M}\right)
$$

and

$c_{k+1}=G\left(d_{k}\right)-C\left(y_{N+k-1}-z_{M}\right), \quad d_{k+1}=G\left(c_{k}\right)+C\left(y_{N+k-1}-z_{M}\right), \quad$ for $k \geq 1$. 
Then,

(i) $\left\{c_{k}\right\}$ is monotonically increasing and $\left\{d_{k}\right\}$ is monotonically decreasing, namely,

$$
0<c_{1}<c_{2}<c_{3}<\cdots<c_{k}<\cdots<d_{k}<\cdots<d_{3}<d_{2}<d_{1}<1 .
$$

(ii) $c_{k}<\epsilon_{N+k}<d_{k} \quad$ for $k \geq 1$.

This can be shown by mathematical induction as follows. For $k=1$,

$$
\begin{aligned}
0 & <c_{1}=G(1)-C\left(y_{N-1}-z_{M}\right) \quad \text { (because of (3.7)) } \\
& \left.<G\left(\epsilon_{N}\right)-C\left(y_{N-1}-z_{M}\right) \quad \text { (because } G \text { is decreasing and } 0<\epsilon_{N}<1\right) \\
& \left.<\epsilon_{N+1}<G\left(\epsilon_{N}\right)+C\left(y_{N-1}-z_{M}\right) \quad \text { (because of }(3.6)\right) \\
& <G(0)+C\left(y_{N-1}-z_{M}\right)=d_{1}<1 \quad \text { (because of (3.7)). }
\end{aligned}
$$

So, both (i) and (ii) hold. Suppose the conclusions hold for $k \geq 1$, that is,

$$
0<c_{1}<\cdots<c_{k-1}<c_{k}, \quad d_{k}<d_{k-1}<\cdots<d_{1}<1 \quad \text { and } \quad c_{k}<\epsilon_{N+k}<d_{k} .
$$

It follows from the monotonicity of both $G$ and the sequence $\left\{y_{k}\right\}$ that

$$
\begin{aligned}
& c_{k+1}=G\left(d_{k}\right)-C\left(y_{N+k-1}-z_{M}\right)>G\left(d_{k-1}\right)-C\left(y_{N+k-2}-z_{M}\right)=c_{k}, \\
& d_{k+1}=G\left(c_{k}\right)+C\left(y_{N+k-1}-z_{M}\right)<G\left(c_{k-1}\right)+C\left(y_{N+k-2}-z_{M}\right)=d_{k} .
\end{aligned}
$$

Furthermore,

$$
\begin{aligned}
c_{k+1} & \left.=G\left(d_{k}\right)-C\left(y_{N+k-1}-z_{M}\right)<G\left(\epsilon_{N+k}\right)-C\left(y_{N+k-1}-z_{M}\right) \quad \text { (because of }(3.8)\right) \\
& \left.<\epsilon_{N+k+1}<G\left(\epsilon_{N+k}\right)+C\left(y_{N+k-1}-z_{M}\right) \quad \text { (because of }(3.6)\right) \\
& \left.<G\left(c_{k}\right)+C\left(y_{N+k-1}-z_{M}\right)=d_{k+1} \quad \text { (because of }(3.8)\right) .
\end{aligned}
$$

Hence,

$$
\begin{gathered}
0<c_{1}<\cdots<c_{k}<c_{k+1}, \quad d_{k+1}<d_{k}<\cdots<d_{1}<1 \quad \text { and } \\
c_{k+1}<\epsilon_{N+k+1}<d_{k+1} .
\end{gathered}
$$

We now let $c_{k} \rightarrow c$ and $d_{k} \rightarrow d$. Then $G(c)=d$ and $G(d)=c$. Since $G(\epsilon)$ is monotonically decreasing, $G^{2}=G \circ G$ is monotonically increasing and can have at most one fixed point. So, $c=d$, and consequently $G(\omega)=\omega$, where

$$
\omega=\lim _{k \rightarrow \infty} \epsilon_{k}=\lim _{k \rightarrow \infty} \frac{y_{k}-z_{M}}{y_{k-1}-z_{M}} .
$$

Thus,

$$
\omega=\frac{2(M-m)}{M\left(1+\omega+\sqrt{(1-\omega)^{2}+\frac{4 m(n-m)}{M(n-M)} \omega}\right)}
$$

or, $\omega$ is a solution to the equation

$$
g(x) \equiv \frac{n-M-m}{n-M} x^{3}-x^{2}-x+\frac{M-m}{M}=0 .
$$

There is only one real solution to $(3.9)$ in $(0,1)$, since

$$
\begin{aligned}
g(0) & =\frac{M-m}{M}>0, \\
g(1) & =\frac{n-M-m}{n-M}-1-1+\frac{M-m}{M}=\left(\frac{n-M-m}{n-M}-1\right)+\left(\frac{M-m}{M}-1\right) \\
& =\frac{-m}{n-M}+\frac{-m}{M}=\frac{-m n}{M(n-M)}<0,
\end{aligned}
$$


and

$$
g^{\prime}(x)=3 \frac{n-M-m}{n-M} x^{2}-2 x-1<0 \text { for } x \in(0,1) .
$$

(ii) For $m=M$, from (3.5), $G\left(\epsilon_{k}\right)=0$ for $k \geq 1$; accordingly, from (3.4),

$$
\epsilon_{k+1}=O\left(y_{k+1}-z_{M}\right) \rightarrow 0 \text { as } k \rightarrow \infty .
$$

Thus, the convergence of $\left\{y_{k}\right\}_{k=1}^{\infty}$ to $z_{M}$ is superlinear. Further, from (2.6), the polynomial $p(x)=C\left(x-y_{k+1}\right)^{m}(x-v)^{n-m}$, where $C$ is a constant and $v$ is a certain real number not in $\left(y_{k}, y_{k-1}\right)$, satisfies

$$
\frac{p^{\prime}\left(y_{k}\right)}{p\left(y_{k}\right)}=\frac{f^{\prime}\left(y_{k}\right)}{f\left(y_{k}\right)} \quad \text { and } \quad \frac{p^{\prime}\left(y_{k-1}\right)}{p\left(y_{k-1}\right)}=\frac{f^{\prime}\left(y_{k-1}\right)}{f\left(y_{k-1}\right)} .
$$

That is,

$$
\frac{M}{y_{k}-y_{k+1}}+\frac{n-M}{y_{k}-v}=\frac{M}{y_{k}-z_{M}}+\sum_{i=1}^{n-M} \frac{1}{y_{k}-z^{(i)}}
$$

and

$$
\frac{M}{y_{k-1}-y_{k+1}}+\frac{n-M}{y_{k-1}-v}=\frac{M}{y_{k-1}-z_{M}}+\sum_{i=1}^{n-M} \frac{1}{y_{k-1}-z^{(i)}} .
$$

Or,

$$
\begin{aligned}
\frac{M\left(y_{k+1}-z_{M}\right)}{\left(y_{k}-z_{M}\right)\left(y_{k}-y_{k+1}\right)} & =-\frac{n-M}{y_{k}-v}+\sum_{i=1}^{n-M} \frac{1}{y_{k}-z^{(i)}}, \\
\frac{M\left(y_{k+1}-z_{M}\right)}{\left(y_{k-1}-z_{M}\right)\left(y_{k-1}-y_{k+1}\right)} & =-\frac{n-M}{y_{k-1}-v}+\sum_{i=1}^{n-M} \frac{1}{y_{k-1}-z^{(i)}} .
\end{aligned}
$$

Subtracting (3.13) from (3.12) and dividing the result by $\left(y_{k-1}-y_{k}\right)$, yields

$$
\begin{aligned}
& \frac{M\left(y_{k+1}-z_{M}\right)\left(y_{k-1}+y_{k}-y_{k+1}-z_{M}\right)}{\left(y_{k}-z_{M}\right)\left(y_{k}-y_{k+1}\right)\left(y_{k-1}-z_{M}\right)\left(y_{k-1}-y_{k+1}\right)} \\
& =-\frac{n-M}{\left(y_{k}-v\right)\left(y_{k-1}-v\right)}+\sum_{i=1}^{n-M} \frac{1}{\left(y_{k}-z^{(i)}\right)\left(y_{k-1}-z^{(i)}\right)} .
\end{aligned}
$$

Note that $R^{*}=\sum_{i=1}^{n-M} \frac{1}{\left(z_{M}-z^{(i)}\right)^{2}}$ in (3.2). Hence, for sufficiently large $k$,

$$
0<\frac{\left(y_{k+1}-z_{M}\right)\left(y_{k-1}+y_{k}-y_{k+1}-z_{M}\right)}{\left(y_{k}-z_{M}\right)\left(y_{k}-y_{k+1}\right)\left(y_{k-1}-z_{M}\right)\left(y_{k-1}-y_{k+1}\right)}<\frac{2 R^{*}}{M},
$$

since $\left(y_{k}-v\right)\left(y_{k-1}-v\right)>0$. Furthermore, the left-hand side of (3.12) satisfies

$$
\begin{aligned}
0 & <\frac{M\left(y_{k+1}-z_{M}\right)}{\left(y_{k}-z_{M}\right)\left(y_{k}-y_{k+1}\right)} \\
= & \frac{M\left(y_{k+1}-z_{M}\right)\left(y_{k-1}-y_{k+1}+y_{k}-z_{M}\right)}{\left(y_{k}-z_{M}\right)\left(y_{k}-y_{k+1}\right)\left(y_{k-1}-z_{M}\right)\left(y_{k-1}-y_{k+1}\right)} \cdot \frac{\left(y_{k-1}-z_{M}\right)\left(y_{k-1}-y_{k+1}\right)}{y_{k-1}-y_{k+1}+y_{k}-z_{M}} \\
& <2 R^{*}\left(y_{k-1}-z_{M}\right) \rightarrow 0 \quad \text { as } \quad k \rightarrow \infty .
\end{aligned}
$$


Thus, the right-hand side of (3.12) gives

$$
\frac{n-M}{y_{k}-v} \rightarrow Q=\sum_{i=1}^{n-M} \frac{1}{z_{M}-z^{(i)}} \quad \text { as } \quad k \rightarrow \infty .
$$

Therefore, when $k \rightarrow \infty$, the right-hand side of (3.14) approaches

$$
E \equiv R^{*}-\frac{Q^{2}}{n-M}=\sum_{i=1}^{n-M} \frac{1}{\left(z_{M}-z^{(i)}\right)^{2}}-\frac{1}{n-M}\left(\sum_{i=1}^{n-M} \frac{1}{z_{M}-z^{(i)}}\right)^{2} \geq 0 .
$$

Let

$$
a=\underbrace{(1, \ldots, 1)}_{n-M} \text { and } \quad b=\left(\frac{1}{z_{M}-z^{(1)}}, \ldots, \frac{1}{z_{M}-z^{(n-M)}}\right) .
$$

Then, by the Cauchy-Schwarz inequality, $|\langle a, b\rangle| \leq\|a\|\|b\|$, or,

$$
\frac{1}{n-M}\left(\sum_{i=1}^{n-M} \frac{1}{z_{M}-z^{(i)}}\right)^{2} \leq \sum_{i=1}^{n-M} \frac{1}{\left(z_{M}-z^{(i)}\right)^{2}} .
$$

Hence, $E=0$ in (3.15) implies $z^{(1)}=z^{(2)}=\cdots=z^{(n-M)}$. That is, $f(x)$ has only two distinct zeros: $z^{(1)}$ and $z_{M}$ with multiplicities $n-M$ and $M$, respectively. By (3.10) and (3.11), one can easily see that in this case $y_{2}=z_{M}$ with starting points $y_{0}$ and $y_{1}$. So, the iteration converges in one step. Thus, we only need to consider the case where $E>0$ in (3.15). Now,

$$
\begin{aligned}
& \lim _{k \rightarrow \infty} \frac{y_{k+1}-z_{M}}{\left(y_{k}-z_{M}\right)^{2}\left(y_{k-1}-z_{M}\right)} \\
&=\lim _{k \rightarrow \infty} \frac{\left(y_{k+1}-z_{M}\right)\left(y_{k-1}+y_{k}-y_{k+1}-z_{M}\right)}{\left(y_{k}-z_{M}\right)\left(y_{k}-y_{k+1}\right)\left(y_{k-1}-z_{M}\right)\left(y_{k-1}-y_{k+1}\right)} \\
& \cdot \frac{\left(y_{k}-y_{k+1}\right)\left(y_{k-1}-y_{k+1}\right)}{\left(y_{k}-z_{M}\right)\left(y_{k-1}-y_{k+1}+y_{k}-z_{M}\right)} \\
&= \frac{E}{M} \cdot \lim _{k \rightarrow \infty} \frac{y_{k}-y_{k+1}}{y_{k}-z_{M}} \cdot \frac{1}{\frac{y_{k-1}-y_{k+1}+y_{k}-z_{M}}{y_{k-1}-y_{k+1}}}=\frac{E}{M} \cdot \lim _{k \rightarrow \infty} \frac{\frac{y_{k}-y_{k+1}}{y_{k}-z_{M}}}{1+\frac{y_{k}-z_{M}}{y_{k-1}-y_{k+1}}} \\
&= \frac{E}{M}>0
\end{aligned}
$$

since

$$
\lim _{k \rightarrow \infty} \frac{y_{k}-y_{k+1}}{y_{k}-z_{M}}=1-\lim _{k \rightarrow \infty} \frac{y_{k+1}-z_{M}}{y_{k}-z_{M}}=1
$$

and

$$
\lim _{k \rightarrow \infty} \frac{y_{k}-z_{M}}{y_{k-1}-y_{k+1}}=\lim _{k \rightarrow \infty} \frac{\frac{y_{k}-z_{M}}{y_{k-1}-z_{M}}}{1-\frac{y_{k+1}-z_{M}}{y_{k-1}-z_{M}}}=0 .
$$

By the following lemma, the convergence rate of $\left\{y_{k}\right\}_{k=1}^{\infty}$ to $z_{M}$ is indeed $\sqrt{2}+1$.

Lemma 3.3. Suppose a sequence $\left\{y_{k}\right\}_{k=1}^{\infty}$ converges to $z$. Let $e_{k}=\left|y_{k}-z\right|$. If

$$
e_{k+1}=M_{k} e_{k}^{2} e_{k-1} \quad \text { and } \quad \lim _{k \rightarrow \infty} M_{k}=M^{*},
$$


where $0<M^{*}<\infty$, then the convergence rate of $\left\{y_{k}\right\}$ is $\sqrt{2}+1$. More precisely,

$$
\lim _{k \rightarrow \infty} \frac{e_{k+1}}{e_{k}^{\sqrt{2}+1}}=\left(M^{*}\right)^{1 / \sqrt{2}} .
$$

Proof. First we assume $M^{*}=1$, that is, $\lim _{k \rightarrow \infty} M_{k}=1$. Let

$$
d_{k}=\ln e_{k+1}-(\sqrt{2}+1) \ln e_{k}, \quad k \geq 1 .
$$

From $e_{k+1}=M_{k} e_{k}^{2} e_{k-1}$ we obtain

$$
\ln e_{k+1}=2 \ln e_{k}+\ln e_{k-1}+\ln M_{k} .
$$

So,

$$
\begin{aligned}
d_{k} & =2 \ln e_{k}+\ln e_{k-1}+\ln M_{k}-(\sqrt{2}+1) \ln e_{k} \\
& =(1-\sqrt{2}) \ln e_{k}+\ln e_{k-1}+\ln M_{k} \\
& =(1-\sqrt{2})\left[\ln e_{k}-(\sqrt{2}+1) \ln e_{k-1}\right]+\ln M_{k} \\
& =(1-\sqrt{2}) d_{k-1}+\ln M_{k} .
\end{aligned}
$$

Since $0>1-\sqrt{2}>-1$ and $\ln M_{k} \longrightarrow 0$ as $k \longrightarrow \infty$, we have $d_{k} \longrightarrow 0$ as $k \longrightarrow \infty$. That is,

$$
\frac{e_{k+1}}{e_{k}^{\sqrt{2}+1}} \longrightarrow 1 \text { as } k \longrightarrow \infty \text {. }
$$

Now, if $M^{*} \neq 1$, let

$$
e_{k}^{\prime}=\sqrt{M^{*}} e_{k} \quad \text { and } \quad M_{k}^{\prime}=\frac{M_{k}}{M^{*}} .
$$

Then,

$$
e_{k+1}^{\prime}=\sqrt{M^{*}} e_{k+1}=\frac{M_{k}}{M^{*}}\left(\sqrt{M^{*}} e_{k}\right)^{2}\left(\sqrt{M^{*}} e_{k-1}\right)=M_{k}^{\prime}\left(e_{k}^{\prime}\right)^{2} e_{k-1}^{\prime}
$$

and

$$
\lim _{k \rightarrow \infty} M_{k}^{\prime}=1 .
$$

So,

$$
\lim _{k \rightarrow \infty} \frac{e_{k+1}^{\prime}}{\left(e_{k}^{\prime}\right)^{\sqrt{2}+1}}=1
$$

and

$$
\lim _{k \rightarrow \infty} \frac{e_{k+1}}{e_{k}^{\sqrt{2}+1}}=\frac{\left(\sqrt{M^{*}}\right)^{\sqrt{2}+1}}{\sqrt{M^{*}}}=\left(M^{*}\right)^{1 / \sqrt{2}} .
$$

\section{Practical consideration on Clusters of Zeros}

In practice, when our quasi-Laguerre iteration is used to approximate a real zero $x^{*}$ of a real polynomial $f$, the multiplicity of $x^{*}$ is, in general, not revealed ahead of time. Therefore, one can only use $m=1$ in (2.10) or (2.11) which may cause slow convergence when the multiplicity of $x^{*}$ is larger than 1. Even when $x^{*}$ is simple, slow convergence may still occur when $x^{*}$ leads a cluster of close zeros of $f$. To overcome this difficulty, we propose the following procedure, which works very efficiently in practice:

By Theorem 3.2, when the quasi-Laguerre iteration for a simple zero $(m=1)$ is used to approximate an $M$-fold zero $x^{*}(M \geq 2)$, or a cluster of $M$ simple zeros, 
of a polynomial $f$ of degree $n$, it converges linearly with a ratio $q_{n, M}$ which is the unique solution in $(0,1)$ of

$$
\frac{n-M-1}{n-M} x^{3}-x^{2}-x+\frac{M-1}{M}=0 .
$$

The following theorem gives the properties of $q_{n, M}$ for $M \geq 2$ and $n \geq M+1$.

Theorem 4.1. (i) For a fixed $M, q_{n, M}$ increases as $n$ increases $(n>M)$.

(ii) For a given $n, q_{n, M} \geq q_{n, 2}$ if $2 \leq M \leq n-2$.

(iii) When $M=n-1$, then $q_{n, n-1}$ increases as $n$ increases $(n \geq 3)$.

Proof. (i) For a fixed $M$, write

$$
g_{n}(x) \equiv \frac{n-M-1}{n-M} x^{3}-x^{2}-x+\frac{M-1}{M} .
$$

Then, when $n_{1}>n_{2}$,

$$
g_{n_{1}}(x)-g_{n_{2}}(x)=\left(\frac{1}{n_{2}-M}-\frac{1}{n_{1}-M}\right) x^{3}>0 \quad \text { for } x \in(0,1) .
$$

So, $q_{n_{1}, M}>q_{n_{2}, M}$.

(ii) For a given $n$, write

$$
g_{M}(x) \equiv \frac{n-M-1}{n-M} x^{3}-x^{2}-x+\frac{M-1}{M} .
$$

When $2 \leq M \leq \frac{n}{2}$, then

$$
\begin{aligned}
g_{M}(x)-g_{2}(x) & =\left(\frac{1}{n-2}-\frac{1}{n-M}\right) x^{3}+\frac{1}{2}-\frac{1}{M} \geq \frac{1}{2}+\frac{1}{n-2}-\frac{1}{M}-\frac{1}{n-M} \\
& =\frac{n}{2(n-2)}-\frac{n}{M(n-M)} \geq 0 \quad \text { for } x \in(0,1) .
\end{aligned}
$$

So, $q_{n, M} \geq q_{n, 2}$.

If $\frac{n}{2}<M \leq n-2$, then $2 \leq n-M<\frac{n}{2}$, and hence, $q_{n, n-M} \geq q_{n, 2}$. On the other hand,

$$
g_{M}(x)-g_{n-M}(x)=\left(\frac{1}{n-M}-\frac{1}{M}\right)\left(1-x^{3}\right)>0 \quad \text { for } x \in(0,1) .
$$

Then $q_{n, M}>q_{n, n-M}$. Thus, $q_{n, M}>q_{n, n-M} \geq q_{n, 2}$.

(iii) If $M=n-1$, write

$$
g_{n}(x) \equiv \frac{n-M-1}{n-M} x^{3}-x^{2}-x+\frac{M-1}{M}=-x^{2}-x+1-\frac{1}{n-1} .
$$

Then, when $n_{1}>n_{2}$,

$$
g_{n_{1}}(x)-g_{n_{2}}(x)=\frac{1}{n_{2}-1}-\frac{1}{n_{1}-1}>0 \quad \text { for } x \in(0,1) .
$$

So, $q_{n_{1}, n_{1}-1}>q_{n_{2}, n_{2}-1}$.

For $n \geq 3$ and $M \geq 2$, by Theorem 4.1 (iii),

$$
q_{n, M} \geq q_{3,2} \quad \text { when } M=n-1,
$$

and by Theorem 4.1 (i), (ii),

$$
q_{n, M} \geq q_{n, 2} \geq q_{3,2} \quad \text { when } M \leq n-2 .
$$


In any case, $q_{3,2}$, the zero of $x^{2}+x-0.5=0$ in $(0,1)$, is the smallest linear ratio for multiple zeros. Let $x^{(1)}, x^{(2)}, \ldots, x^{(k)}, \ldots$ be the monotone sequence of the quasi-Laguerre iterates. When $\left\{x^{(k)}\right\}$ converges superlinearly, then

$$
q_{k} \equiv \frac{\left|x^{(k+1)}-x^{(k)}\right|}{\left|x^{(k)}-x^{(k-1)}\right|} \longrightarrow 0 \quad \text { as } k \rightarrow \infty,
$$

so, $q_{k}$ should be smaller than $q_{3,2}$ when $k$ is sufficiently large. Therefore, when $q_{k}>q_{3,2}$, linear convergence seems apparent. In this case, for sufficiently large $k$,

$$
\frac{\left|x^{(k+1)}-x^{*}\right|}{\left|x^{(k)}-x^{*}\right|} \approx q_{n, M} \quad \text { or } \quad\left|x^{(k+1)}-x^{*}\right| \approx q\left|x^{(k)}-x^{*}\right| \quad \text { where } q \equiv q_{n, M},
$$

and

$$
q_{k}=\frac{\left|x^{(k+1)}-x^{(k)}\right|}{\left|x^{(k)}-x^{(k-1)}\right|}=\frac{\left|x^{(k)}-x^{*}\right|-\left|x^{(k+1)}-x^{*}\right|}{\left|x^{(k-1)}-x^{*}\right|-\left|x^{(k)}-x^{*}\right|} \approx \frac{(1-q)\left|x^{(k)}-x^{*}\right|}{(1-q)\left|x^{(k-1)}-x^{*}\right|} \approx q .
$$

In practice, the $q_{k}$ 's can be very close to $q$ after several iterations. If $q_{j}=q$ for $j \geq k$, then

$$
\left|x^{(j+1)}-x^{*}\right|=q\left|x^{(j)}-x^{*}\right| \text { for } j \geq k,
$$

and

$$
x^{*}=x^{(k)}+\frac{1}{1-q}\left(x^{(k+1)}-x^{(k)}\right) .
$$

So, at the $k$ th iterate $x^{(k)}$, if linear convergence is revealed, namely $q_{k}>q_{3,2}$, then instead of performing the regular quasi-Laguerre iteration

$$
x^{(k+1)}=x^{(k)}+\delta_{k},
$$

where $\delta_{k}$ is the correction $x^{(k+1)}-x^{(k)}$ to $x^{(k)}$ calculated by the quasi-Laguerre iteration with $m=1$, one can accelerate the iteration by setting

$$
x^{(k+1)}=x^{(k)}+\frac{\delta_{k}}{1-q_{k}} .
$$

To illustrate the efficiency of this acceleration device, consider a Wilkinson matrix $W_{99}^{+}$with dimension $n=99$. This is a symmetric tridiagonal matrix whose eigenvalues are mostly pairs of numerically indistinguishable real numbers $[5, \mathrm{pp}$. $308-309]$. Let $f$ be the characteristic polynomial of $W_{99}^{+}$; namely,

$$
f(z)=\operatorname{det}\left(W_{99}^{+}-z I\right) .
$$

The zeros of $f$ are the eigenvalues of $W_{99}^{+}$. The pair $z_{1}=11.0+5 \times 10^{-15}$ and $z_{2}=11.0-5 \times 10^{-15}$ is a pair of close zeros of $f$. To approximate $z_{1}$, the starting points $x^{(0)}=11.5$ and $x^{(1)}=11.27$ (one step Laguerre's iteration from $x^{(0)}$ ) are used. For $k=1,2, \ldots, \delta_{k}=x^{(k+1)}-x^{(k)}$ is evaluated using the quasi-Laguerre formula with $m=1$. If $q_{k}<q_{3,2}$, then most likely $z_{1}$ is neither a multiple zero nor a member of any cluster. Then $x^{(k+1)}$ is accepted and we continue onto the next iterate. Otherwise, let $c=x^{(k)}+\frac{\delta_{k}}{1-q}$ and check for possible overshoot by evaluating the Sturm sequence at $c$. If $c$ overshoots, then reset $c=x^{(k)}+\frac{\delta_{k}\left(1-q^{l}\right)}{1-q}$ for $l=8,4,2,1$ until overshooting disappears. Then $c$ is accepted as $x^{(k+1)}$ and we continue onto the next iterate. 
TABLE 1. Acceleration of the quasi-Laguerre iteration for $W_{99}^{+}$. For each number in the table, the digits before a space are correct

\begin{tabular}{|c|c|c|c|}
\hline$k$ & $x^{(k)}$ (original) & $q_{k}=\frac{\left|x^{(k+1)}-x^{(k)}\right|}{\left|x^{(k)}-x^{(k-1)}\right|}$ & $x^{(k)}$ (accelerated) \\
\hline 1 & 11.500000000000000 & & 11. 500000000000000 \\
\hline 2 & 11.270700712327294 & & 11. 270700712327294 \\
\hline 3 & 11.149428542073966 & 0.706618 & 11.013287361699255 \\
\hline 4 & 11.063735435125402 & 0.437604 & 11.000124299930121 \\
\hline 5 & 11.026235830973979 & 0.417533 & 11.000061856842477 \\
\hline 6 & 11.010578521008206 & 0.403427 & 11.000000198368395 \\
\hline 7 & 11.004261938886133 & 0.403112 & 11.000000099023529 \\
\hline 8 & 11.001715645778917 & 0.402555 & 11.000000000012077 \\
\hline 9 & 11.000690622605815 & 0.402556 & 11.000000000006040 \\
\hline 10 & 11.000277993423158 & 0.402527 & 11.000000000002304 \\
\hline 11 & 11.000111899071095 & 0.402525 & 11.000000000000156 \\
\hline 12 & 11.000045041905516 & 0.402523 & 11.000000000000064 \\
\hline 13 & 11.000018130368451 & 0.402522 & \\
\hline 14 & 11.000007297872838 & 0.402522 & \\
\hline 15 & 11.000002937554150 & 0.402522 & \\
\hline 16 & 11.000001182429996 & 0.402522 & \\
\hline 17 & 11.000000475954003 & 0.402522 & \\
\hline 18 & 11.000000191581922 & 0.402522 & \\
\hline 19 & 11.000000077115924 & 0.402522 & \\
\hline 20 & 11.000000031040850 & 0.402522 & \\
\hline 21 & 11.000000012494622 & 0.402522 & \\
\hline 22 & 11.000000005029360 & 0.402522 & \\
\hline 23 & 11.000000002024429 & 0.402522 & \\
\hline 24 & 11.000000000814877 & 0.402522 & \\
\hline 25 & 11.000000000328006 & 0.402522 & \\
\hline 26 & 11.000000000132029 & 0.402520 & \\
\hline 27 & 11.000000000053145 & 0.402521 & \\
\hline 28 & 11.000000000021393 & 0.402526 & \\
\hline 29 & 11.000000000008612 & 0.402521 & \\
\hline 30 & 11.000000000003467 & 0.402477 & \\
\hline 31 & 11.000000000001396 & 0.401680 & \\
\hline 32 & 11.000000000000565 & 0. 399604 & \\
\hline 33 & 11.000000000000233 & 0.381937 & \\
\hline 34 & 11.000000000000107 & 0.305425 & \\
\hline
\end{tabular}

Table 1 gives the comparison between the original quasi-Laguerre iteration and the accelerated quasi-Laguerre iteration. Notice that the $q_{k}$ 's in the third column of Table 1 are identical up to six digits with $q=q_{99,2} \approx 0.402522$.

\section{REFERENCES}

1. Qiang Du, Ming Jin, T.Y. Li and Z. Zeng, Quasi-Laguerre iteration in solving symmetric tridiagonal eigenvalue problems, to appear: SIAM J. Sci. Comput.

2. L. V. Foster, Generalizations of Laguerre's method: lower order methods, preprint.

3. W. Kahan, Notes On Laguerre's Iteration, preprint, University of California, Berkeley (1992). 
4. T. Y. Li and Z. Zeng, The Laguerre iteration in solving the symmetric tridiagonal eigenproblem - revisited, SIAM J. Sci. Comput. 15 (1994), 1145-1173. MR 95h:65024

5. J. H. Wilkinson, The algebraic eigenvalue problem, Clarendon Press, Oxford, England, 1965. MR 32:1894

Department of Mathematics, Michigan State University, East Lansing, Michigan 48824

E-mail address: du@math.msu.edu

Department of Mathematics, Michigan State University, East Lansing, Michigan 48824

Current address: Department of Mathematics, Lambuth University, Jackson, Tennessee 38301

E-mail address: jinm66@usit.net

Department of Mathematics, Michigan State University, East Lansing, Michigan 48824

E-mail address: li@math.msu.edu

Department of Mathematics, Northeastern Illinois University, Chicago, Illinois 60625

E-mail address: uzzeng@uxa.ecn.bgu.edu 